\title{
Effectiveness of Reactive Power Capability of Photo Voltaic Inverters to Maintain Voltage Profile in a Residential Distribution Feeder
}

\author{
M. N. Kabir, Student Member, IEEE, Y. Mishra, Member, IEEE
}

\begin{abstract}
Large penetration of rooftop PVs has resulted in unacceptable voltage profile in many residential distribution feeders. Limiting real power injection from PVs to alleviate over voltage problem is not feasible due to loss of green power and hence corresponding revenue loss. Reactive capability of the PV inverter can be a solution to address over voltage and voltage dip problems to some extent. This paper proposes an algorithm to utilize reactive capability of $\mathrm{PV}$ inverters and investigate their effectiveness for voltage improvement based on $R / X$ ratio of the feeder. The length and loading level of the feeder for a particular $R / X$ ratio to have acceptable voltage profile is also investigated. This can be useful for suburban design and residential distribution planning. Furthermore, coordination among different PVs using residential smart meters via a substation based controller is also proposed.
\end{abstract}

Index Terms-Photo Voltaic (PV), Peak demand, Rural networks, Voltage profile.

\section{INTRODUCTION}

$\mathrm{T}$ HERE is significant contribution of electricity generation from rooftop Photo Voltaic (PV) in recent years [1]. The feasibility of small scale PV integrations in urban and rural areas has been thoroughly demonstrated in [2]-[4]. Government incentives combined with public awareness for green technology has resulted in unprecedented growth of rooftop PV modules in residential premises. The penetration of PVs increases the service reliability and defers the need for immediate investment in grid reinforcement [5]. However, high level penetration of PV brings several challenges among which voltage rise is the most prominent [6]-[9]. In [10], an approach is proposed to control the voltage using transformer tap settings. The main limiting factor of this approach is the inability of frequent tap changing and finding an optimal setting. Particular focus has been given to the real-time setting of the On-Load Tap Changer (OLTC) of distribution substations [11]. After instant demand forecasting and measurements of power injected by PVs, it is possible to determine OLTC settings to keep the voltage within limits. This requires reliable communication link between PV

Manuscript received October 31, 2013.

Md Nayim Kabir (email: mn.kabir@qut.edu.au) and Yateendra Mishra (email: yateendra.mishra@qut.edu.au) are with the School of Electrical customers and system operators [11]-[13]. Another way of keeping the acceptable feeder voltage is to limit the PV injection [6], [10]. Such capacity limitation hinders the effective utilization of green energy.

Though not permitted under existing standards of PV interconnection, feeder voltage control using the reactive capability of PV inverters can be a useful method to avoid voltage violations. In this paper, an algorithm for maintaining voltage profile by reactive power control is shown for two scenarios, urban case (low $\mathrm{R} / \mathrm{X}$ ratio) and rural case (considering Single Wire Earth Return-SWER line with comparatively higher $\mathrm{R} / \mathrm{X}$ ratio). References [14]-[18] give some insight on the reactive power control but they are developed mainly for Medium Voltage (MV) and High Voltage (HV) networks. Also, line characteristics such as feeder length and line resistivity are not considered.

Voltage control capability of rooftop PV inverters can be affected by feeder length and its $\mathrm{R} / \mathrm{X}$ ratio. The focus of this paper is to quantify the reactive power capability of PV inverters to maintain the acceptable voltage limit in the residential distribution feeder. An algorithm to use the reactive capability of PV inverter is also developed. Feasibility of incorporating the developed control algorithm in a distribution network is briefly discussed. This paper is organized as follows: Section II introduces an algorithm for utilizing reactive power capability of PV inverters. It also mentions about how the developed algorithm can be incorporated in distribution system with Smart Meters and Home Area Network. Section III describes the residential system, PV generation and load profile used in this study. Section IV describes effectiveness of reactive power capability of $\mathrm{PV}$ inverters for urban and rural Cases. The impact of line resistivity and feeder distances are discussed and quantified with results in Section V and VI respectively followed by discussions in Section VII.

\section{REACTIVE POWER CAPABILITY OF PV INVERTERS AND COMMUNICATION NETWORK}

The reactive power capability of PV inverter can be utilized for voltage control. If the apparent power capability and instantaneous real power generated from an inverter are $S$ and

Engineering and Computer Science, Queensland University of Technology (QUT), Brisbane, QLD, Australia-4000) 
$P_{G}$ (where $S>P_{G}$ ) respectively, then the reactive capability range of PV inverter is given by $\left|Q_{G}\right| \leq \sqrt{ }\left(S^{2}-P_{G}{ }^{2}\right)$. Thus, for a $6 \mathrm{kVA}$ inverter, at $0.8 \mathrm{PF}$, maximum allowable real power $\left(P_{G}\right)$ is 4.8 $\mathrm{kW}$. Maximum reactive power $\left(Q_{G}\right)$ generation is $3.6 \mathrm{kVAR}$ and $6 \mathrm{kVAR}$ with maximum and minimum $P_{G}$ generation respectively. In this paper, reactive power control algorithm is developed in such a manner that PV inverters absorb reactive power during solar radiation time and supplies reactive power during peak load demand time to address the voltage violation problem. This can be achieved by controllers communicating with smart meters.

Each residential premise can be equipped with advanced metering system such as Home Area Network (HAN) interfaced with PV inverter controlled by Smart Meters (SM). HAN is an in-house network and have inter-operation among all the connected devices. It ensures bi-directional secured communication among the devices to automate the proposed control algorithm. Appropriate authentication mechanism is an integrated part of HAN which allows only the recognized devices to connect the network [19]. HAN is configured within an individual residential premise with the functionality of i) inhouse energy management; ii) use the reactive capability of PV inverter using the developed control algorithm.

Successful operation of the proposed algorithm requires coordination and communication between HAN system of all the houses as well as with the substation controller. Several HAN system can communicate with each other through SMs. The communication medium of HAN as well as SMs can be wired (power line cable) or wireless network (cellular networks). Use of existing power line cable as communication medium, known as Power Line Communication (PLC) [20], is a promising technique for inter-substation communications. An extensive and pervasive network is already developed for all the end users; and therefore the deployment cost in networking infrastructure, like dedicated cables or antennas can be omitted. In fact, the communication requirement for HAN and/or SMs is protocol dependant. For example, in case of fault management, the objective is to instantly and accurately identify the fault feeders and trigger the protection action [21], [22]. One of the key qualities of service for such protective actions is "Message Delivery Delay", which makes PLC not favorable for HAN application. In the proposed algorithm, whereas, the response time of $2 \mathrm{~s}-30$ s is acceptable and hence PLC can be used in HAN application and in sending/receiving information between substation controller and individual SMs.

The centralized controller at the substation level communicates with the SM of each house to determine the necessary reactive power to be absorbed/ supplied from the PV inverter. SM of each house carries this message signal to corresponding PV inverter through HAN system. In this way, the proposed reactive support algorithm can be easily deployed in the distribution system.

Proposed algorithm using the PLC to ensure communication between substation controller-individual SM and individual SM-corresponding PV inverter is summarised in Fig. 1. The algorithm runs in an hourly basis.

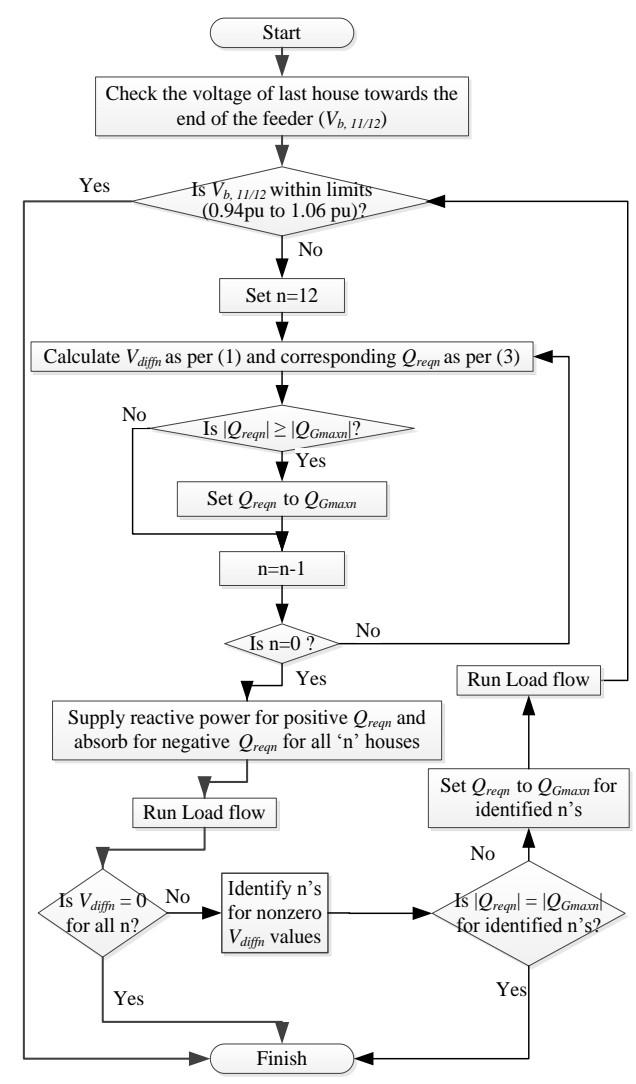

Fig. 1. Reactive Power Control Flow-chart

- $1^{\text {st }}$ step: At substation controller, voltage at each house is calculated from a load flow without any reactive power injection or absorption. Necessary information (PV generation, load demand, etc.) to run the load flow is collected from the individual SMs at each house. These voltages identify whether any house violates the voltage profile in normal situation at that particular time. For the initial checking it only considers the voltage of last house. For a radial line, all other houses do not have any violation if voltage at last house is within limit.

- $2^{\text {nd }}$ step: If voltage violation exists, difference voltage $\left(V_{\text {diffn }}\right)$ is calculated using (1) and then required reactive power $\left(Q_{\text {reqn }}\right)$ to minimize this voltage difference is calculated using (3) for each of the houses at substation controller.

- $3^{\text {rd }}$ step: For each house the substation controller checks whether $Q_{\text {reqn }}$ exceeds the maximum inverter capacity $\left(Q_{G \operatorname{maxn}}\right)$ of a particular house. If $Q_{\text {reqn }}$ exceeds $Q_{\text {Gmaxn }}$ then $Q_{\text {reqn }}$ is set to the maximum inverter capacity i. e. $Q_{\text {Gmaxn }}$. Same steps are followed for all the 12 houses.

- $4^{\text {th }}$ step: After determining $Q_{\text {reqn }}$ for all the houses a load flow is run to check the voltages of all houses again by the controller.

- $5^{\text {th }}$ step: If still the voltages are not within the limit, critical house nodes (houses with violated voltage profile) are identified. $Q_{\text {reqn }}$ is set to $Q_{\text {Gmaxn }}$ for these particular houses. And for other houses new $Q_{\text {reqn }}$ is calculated using (3). Again the load flow is run and voltage is checked for all the houses by substation controller.

- $6^{\text {th }}$ step: This iterative process runs until all the houses voltages fall within acceptable range or reach the maximum 
inverter capacity though acceptable voltage profile is not yet achieved for all (this is the situation in rural case). Determined $Q_{\text {reqn }}$ is sent to corresponding SMs by the substation controller. SMs of each house communicate with PV inverters using HAN system to perform the reactive support.

Equations for determining $V_{\text {diff }}$ and $Q_{\text {reqn }}$ are as follows:

$$
V_{\text {diffin }}=V_{a n}-V_{b n}
$$

Where $V_{b n}$ is the house bus voltage for $n^{\text {th }}$ house and $V_{a}$ is acceptable bus voltage as in (2).

$$
V_{a n}=\left\{\begin{array}{cl}
1.06 p u, & \text { if } V_{b n} \geq 1.06 p u \\
0.94 p u, & \text { if } V_{b n} \leq 0.94 p u \\
V_{b n}, & \text { otherwise }
\end{array}\right.
$$

Where, $k$ is a constant. Based on the sign of difference voltage $V_{\text {diff }}$ (positive or negative), decision on reactive power injection or absorption from PV inverter is made for each bus. Negative value indicates the situation when house voltage $V_{b n}$ $\geq 1.06 \mathrm{pu}$, so reactive power is absorbed. Reactive power is supplied when the house voltage $V_{b n} \leq 0.94 \mathrm{pu}$.

\section{SYSTEM DESCRIPTION}

A particular test network is considered for simulating the impact of feeder length and $\mathrm{R} / \mathrm{X}$ ratio of line impedances on reactive control (

Fig. 2). The test network consists of an $11 \mathrm{kV} / 240 \mathrm{~V}$ transformer supplying electricity to 12 houses in a $120 \mathrm{~m}$ long overhead residential feeder. Different set of line parameters are used to investigate the impact of feeder length and $\mathrm{R} / \mathrm{X}$ ratio of line impedances. In an urban scenario, distance between backbone buses, $F_{d}$, and corresponding houses, $d$, are 20 meters each. R/X ratio is considered unity for the overall urban network. Whereas, in a rural scenario (Single Wire Earth Return (SWER)), distance between backbone buses, $F_{d}$, and corresponding houses, $d$, are 100 meters and 20 meters respectively. $\mathrm{R} / \mathrm{X}$ ratio is considered to be around 5.6. These parameter values are taken from an Australian distribution company.

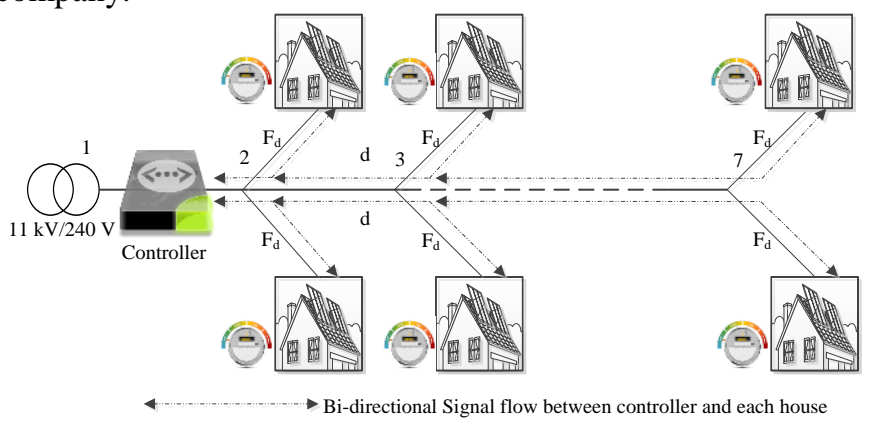

Fig. 2. Model of the test system

A typical summer peak load profile for a particular house is taken from one of the Australian Distribution Company's Annual Report which reaches maximum load of $3 \mathrm{~kW}$ at $8 \mathrm{pm}$. The PV generation profile is assumed to have a normal distribution and starts from 6 am and reaches peak of $6 \mathrm{~kW}$ at 12 noon and then gradually dies out at $6 \mathrm{pm}$. Identical PV generation and load profile (a lagging Power Factor (PF) of 0.8) is assumed for all the houses as shown in Fig. 3.

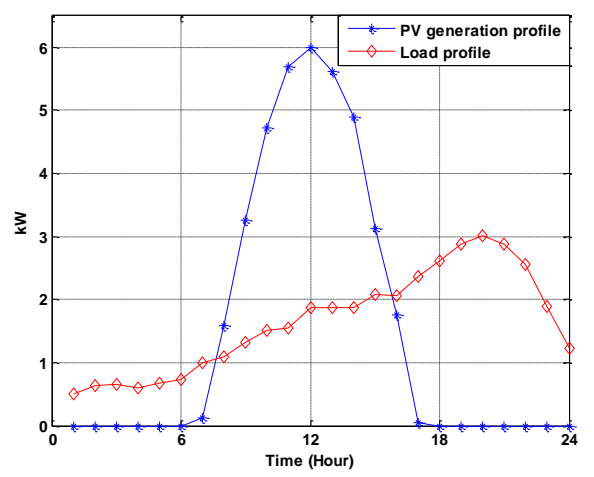

Fig. 3. PV generation and load profile for a peak day

\section{EFFECTIVENESS OF REACTIVE POWER CAPABILITY OF PV INVERTERS FOR URBAN AND RURAL CASES}

MATLAB is used for all the simulation results presented in this paper. For a system shown in

Fig. 2 and the assumed PV generation and load profile as in Fig. 3, voltage profile across the feeder is investigated. As two houses are connected with same backbone bus and have the same characteristics, only 6 houses are plotted for 24 hours.

\section{A. Without reactive capability of $P V$ inverters}

In normal situation (without using the reactive capability of PV inverter), voltage profile of all houses are shown in Fig. 4 (urban case) and Fig. 5 (rural case). Grid connected PVs are considered to operate at unity PF with Maximum Power Point Tracking (MPPT) algorithm.

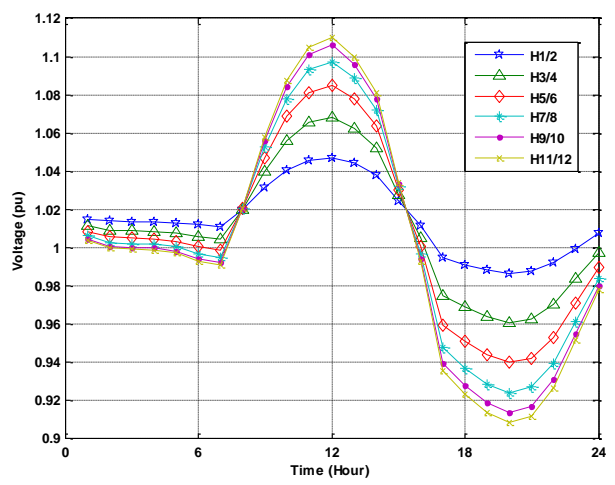

Fig. 4. 24 Hour Voltage Profile for urban case in normal situation

From Fig. 4 and Fig. 5, it is evident that the voltage at the end of the feeder is extremely high when PV generation is peak. House No\# 11 and 12, (H-11/12) has $1.11 \mathrm{pu}$ and $1.13 \mathrm{pu}$ voltages at 12 noon for urban and rural case respectively (LV feeder voltage allowable range is $\pm 6 \%$ [9]). All the houses (except first 2 houses for both the cases) exceed their voltages from the highest allowable range $1.06 \mathrm{pu}$ when load $(1.875 \mathrm{~kW})$ is much lower than the peak generation $(6 \mathrm{~kW})$. On the other hand, due to absence of PV generation at peak demand or evening time, voltage profile gets a dip and all the houses (except first 6 houses for urban case and first 4 houses for rural case) cross the lower limit. Fig. 5 shows even worse voltage profile (higher overvoltage and higher voltage dip) for rural 
case which has longer feeder and higher $\mathrm{R} / \mathrm{X}$ value in line impedances. In other way it can be said that networks with longer feeder and/or higher $\mathrm{R} / \mathrm{X}$ value in line impedances have worse voltage profile.

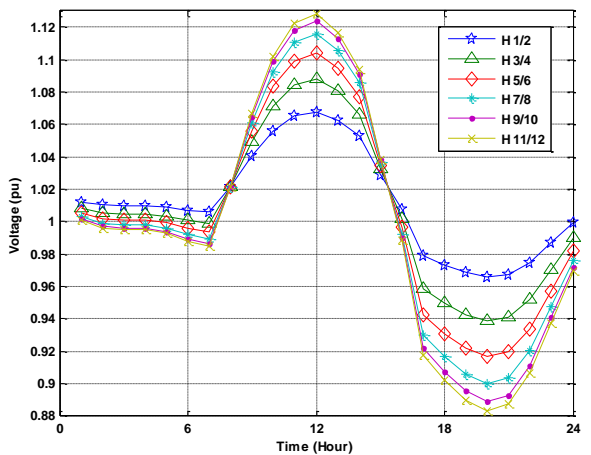

Fig. 5. 24 Hour Voltage Profile for rural case in normal situation

\section{$B$. With reactive capability of $P V$ inverters}

The reactive power capability of PV inverter is utilized for the voltage control of all the houses as described in Section III. Fig. 6 shows the voltage profile of all houses and corresponding $\mathrm{PV}$ inverter reactive power injection/absorption for urban case. Voltage of House No\# 11 and 12 (H-11/12) during peak PV generation has been reduced below 1.06 pu (Fig. 6) using the reactive capability of the $\mathrm{PV}$ inverter. H11/12 voltage was 1.11 pu (Fig. 4) without reactive absorption. Similarly, during evening where there is no PV generation and loading level reaches the peak, the voltage dip is improved by the reactive power injection from PV inverters. Therefore, using PV inverter reactive capability, the house voltages can be kept within acceptable limits in the urban case after applying the developed algorithm.

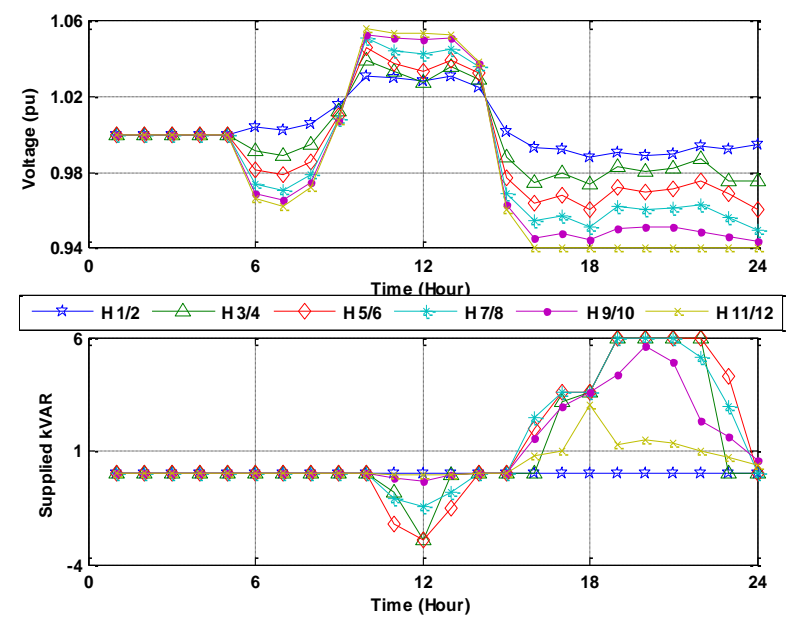

Fig. 6. Voltage profile and PV inverter reactive power compensation in urban case

Voltage profile of all the houses across SWER lines and their PV inverter reactive power injection/absorption for the rural case is shown in Fig. 7. Voltage profile shows violation for the houses particularly in voltage dip (during peak loading and no $\mathrm{PV}$ generation), even after using the maximum capability of PV inverter. Therefore, the conclusion can be made that using the reactive capability of individual $\mathrm{PV}$ inverter may not be effective due to higher $\mathrm{R} / \mathrm{X}$ ratio of line in rural scenario. In the following sections the impact of $\mathrm{R} / \mathrm{X}$ ratio and feeder distance will be quantified.

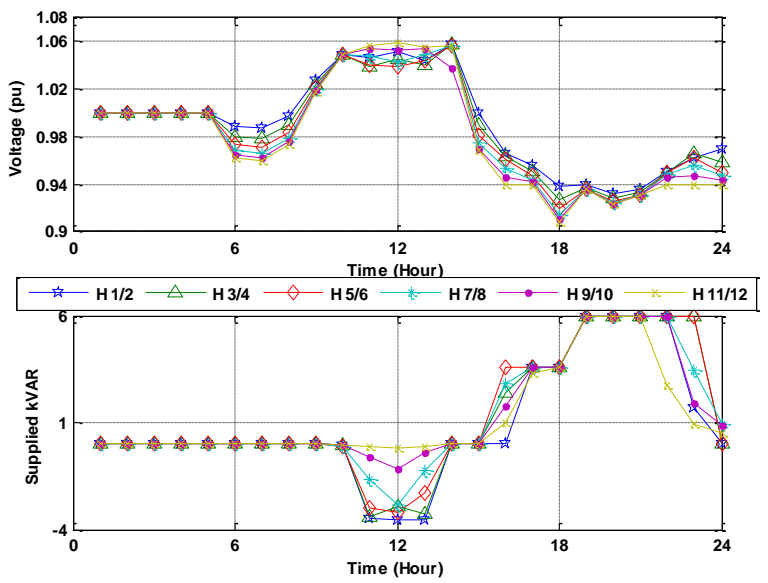

Fig. 7. Voltage profile and PV inverter reactive power compensation for rural case

\section{IMPACT OF LINE RESISTIVITY}

The key focus of this section is to determine a limit of R/X ratio for which $\mathrm{PV}$ inverter reactive power capability is not sufficient to improve the voltage dip during peak loading. $\mathrm{R} / \mathrm{X}$ ratio of the line between feeder bus and adjacent house (shown as $d$ in Fig. 2) is varied from 1 to 8. Peak loading is simulated keeping the injection of reactive power maximum i.e. 3.6 $\mathrm{kVAR}$ considering the real power is not sacrificed/curtailed at any time. $\mathrm{R} / \mathrm{X}$ ratio of backbone feeder is kept unity. Simulation is performed at 0.8 load $\mathrm{PF}$ and $100 \%$ loading in each house. Fig. 8 shows that when $\mathrm{R} / \mathrm{X}$ ratio is higher than 4.5 , voltage goes below lower acceptable range $(0.94 \mathrm{pu})$.

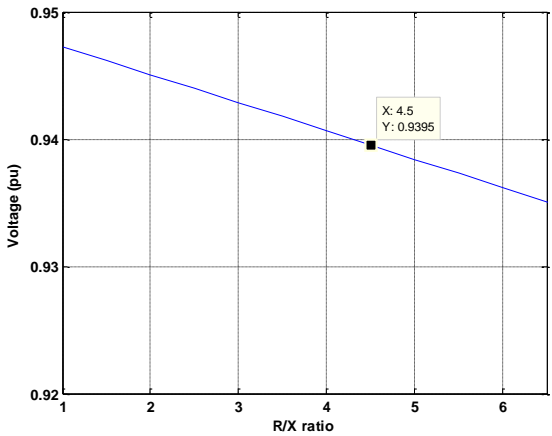

Fig. 8. Voltage level Vs R/X ratio with full loading

A trade off in loading level is required to keep the voltages within acceptable range $(0.94 \mathrm{pu})$ for a feeder whose $\mathrm{R} / \mathrm{X}$ ratio is higher than 4.5. Fig. 9 shows the percentage of feeder loading level with increase in $\mathrm{R} / \mathrm{X}$ ratio for maximum reactive power injection and zero reactive power injection. Steps for determining the permitted loading curve with maximum reactive power injection are shown in Fig. 10. Similar steps are followed for determining the same curve with zero reactive injection from PV inverter. From Fig. 9, it is observed that with higher $\mathrm{R} / \mathrm{X}$ ratio (higher than $4.5 \sim 5$ ), loading should be reduced to $70 \%$ of peak value to keep the voltages in acceptable range. 
Moreover, same reactive injection incurs higher losses in higher resistive lines. Plotting incremental loss (increase in loss with respect to unity $\mathrm{R} / \mathrm{X}$ ) with $\mathrm{R} / \mathrm{X}$ ratio variation is also shown in Fig. 9. After certain $\mathrm{R} / \mathrm{X}$ ratio (above $4.5 \sim 5$ ) losses are too high (50\% increase).

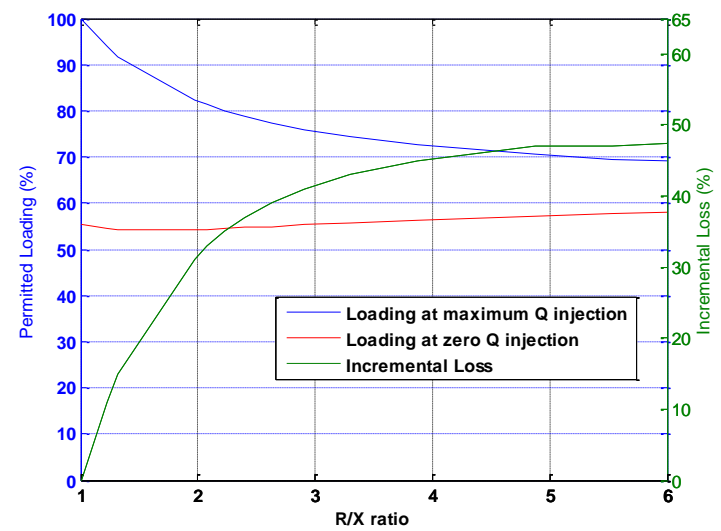

Fig. 9. Permitted loading and incremental loss variation with $\mathrm{R} / \mathrm{X}$ ratio

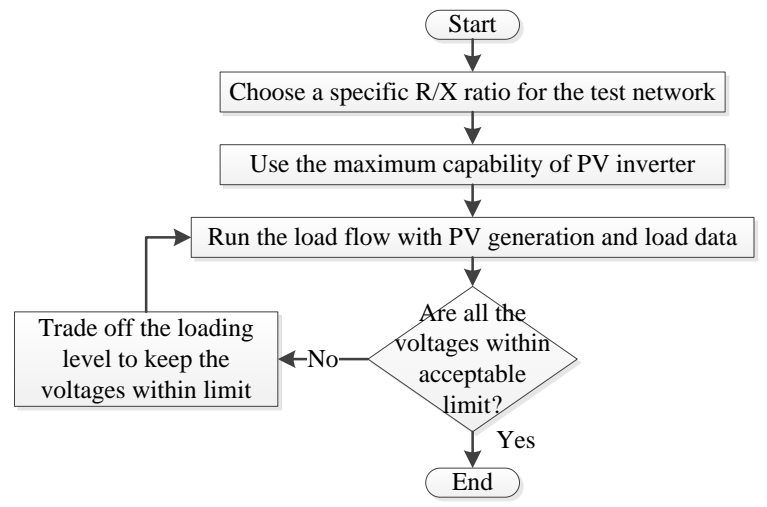

Fig. 10. Steps for getting the permitted loading curve

Therefore it can be concluded that, the effectiveness of PV inverters for voltage control is limited if the $\mathrm{R} / \mathrm{X}$ ratio of the feeder is higher than 4.5 5.

\section{IMPACT OF FEEDER DisTANCE}

Impact of backbone feeder distance ( $F_{d}$ in Fig. 2$)$ on the effectiveness of $\mathrm{PV}$ inverter reactive power capability to maintain the acceptable voltage profile is investigated in this section. In previous section, only distance between feeder bus and adjacent house ( $d$ in Fig. 2 ) is increased and $F_{d}$ is kept constant. Here, $F_{d}$ is variable to find a relationship between $F_{d}$ and $\mathrm{R} / \mathrm{X}$ ratio, if any, to maintain an acceptable voltage profile. $\mathrm{PV}$ inverter reactive capability is kept maximum i.e. 3.6 kVAR, assuming that the real power generation from PV is not sacrificed/curtailed at any time.

For a particular $F_{d}$, load flow is performed to observe feeder voltages and $\mathrm{R} / \mathrm{X}$ ratio is reduced if voltage is not acceptable and resolve the load flow. This is continued until acceptable feeder voltages are achieved. This procedure of finding $\mathrm{R} / \mathrm{X}$ ratio for a particular $F_{d}$ is repeated for all $F_{d}$ 's ranging from 7 to 56 meters. $\mathrm{R} / \mathrm{X}$ ratio is kept within the range $1 \sim 11$, which is acceptable for a rural distribution network.
TABLE I. RELATION BETWEEN $F_{D}$ AND R/X RATIO OF THE FEEDER BACKBONE FOR AN ACCEPTABLE FEEDER VOLTAGE PROFILE

\begin{tabular}{|l|l|l|}
\hline $\begin{array}{l}\mathbf{F}_{\mathbf{d}} \\
(\mathbf{m e t e r s})\end{array}$ & $\mathbf{R} / \mathbf{X}$ ratio & $\begin{array}{l}\left(\boldsymbol{F}_{\boldsymbol{d}}\right) *(\mathbf{R} / \mathbf{X} \text { ratio }) \\
(\text { meters })\end{array}$ \\
\hline 7 & 11 & 77 \\
\hline 14 & 5.57 & 77.97 \\
\hline 16 & 4.89 & 78.24 \\
\hline 17 & 4.55 & 77.35 \\
\hline 18 & 4.52 & 81.38 \\
\hline 19 & 4.4 & 83.6 \\
\hline 22 & 3.7 & 81.4 \\
\hline 28 & 2.78 & 77.81 \\
\hline 56 & 1.39 & 77.97 \\
\hline
\end{tabular}

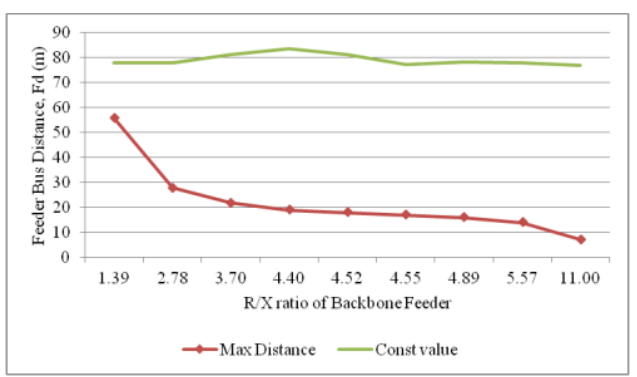

Fig. 11. $\left(F_{d}\right) *(\mathrm{R} / \mathrm{X}$ ratio $)$ plotted against $\mathrm{R} / \mathrm{X}$ ratio of the feeder backbone

TABLE I shows the relationship between $F_{d}$ and R/X ratio for acceptable voltage profile. It is interesting to observe that $\left(F_{d}\right) *(\mathrm{R} / \mathrm{X}$ ratio $)$ is more or less same for all simulations except for $\mathrm{R} / \mathrm{X}$ ratio of 3.7 to $4.5 .\left(F_{d}\right) *(\mathrm{R} / \mathrm{X}$ ratio $)$ value is slightly higher (81 83) in this range but within $5 \%$ of the other values of $\left(F_{d}\right) *(\mathrm{R} / \mathrm{X}$ ratio $)$. Fig. 11 shows the value of $\left(F_{d}\right) *(\mathrm{R} / \mathrm{X}$ ratio $)$ and its variation with $\mathrm{R} / \mathrm{X}$ ratio of the feeder.

From the analysis a significant conclusion can be made that when distance between adjacent buses in the backbone feeder $\left(F_{d}\right)$ is increased, the $\mathrm{R} / \mathrm{X}$ ratio of the backbone feeder should be reduced so that their multiplication is kept nearly constant for acceptable voltage profile. This can be relevant for rural network distribution planning.

\section{DISCUSSION}

Voltage violation problem with higher $\mathrm{R} / \mathrm{X}$ ratio can be addressed by using Distributed STATic COMpensator (DSTATCOM). Reactive power injection or absorption can be done by placing a D-STATCOM in the optimal location of the feeder. Optimum D-STATCOM sizing to eliminate voltage violations for the test network is determined running load flow iteratively by placing it at the $5^{\text {th }}$ bus. Researchers already identified the optimal placement of D-STATCOM which is two third of the distance i.e. nearly $70 \%$ of feeder distance [23]. For the given network, $65 \mathrm{kVAR}$ D-STATCOM is required, which needs investment of $\$ 3,500-3,750$ from the Distribution Network Service Provider (DNSP) (assuming cost of \$5055/kVAR for the D-STATCOM [24]). Although investment in D-STATCOM from the DNSP can be a solution to address the voltage violation problem, introduction of Battery Energy Storage (BES) in the distribution feeder may provide long term technical benefit in terms of peak shaving. D-STATCOM can regulate voltage violation with reactive power support only and cannot supply real power during peak demand. Integration of 
BES can be one of the solutions to mitigate voltage violation problem as well as to support the grid with real power.

\section{CONCLUSION}

This paper introduces an algorithm to utilize the reactive power capability of PV inverters and its effectiveness under two different scenarios. Urban scenario has unity $\mathrm{R} / \mathrm{X}$ ratio whereas rural case considers SWER line with $\mathrm{R} / \mathrm{X}$ ratio of 5.6. Simulation results show that reactive power capability of PV inverter can be sufficient for urban case to maintain acceptable voltage profile but rural case would require additional support such as capacitor banks, storage or load curtailment.

Furthermore, the effectiveness of PV inverters for voltage control is also investigated in this paper. For feeders having $\mathrm{R} / \mathrm{X}$ ratio less than 4.5 , reactive power capability of $\mathrm{PV}$ inverter is sufficient for voltage control. Also, it is observed that the product of the distance between adjacent buses in the backbone feeder $\left(F_{d}\right)$ and $\mathrm{R} / \mathrm{X}$ ratio should be maintained nearly constant for acceptable voltage profile. This is relevant for newer suburban design and rural network distribution planning. The solution to maintain acceptable voltage profile for longer feeders and highly resistive lines can be a coordinated control of reactive power and BES. The energy can be stored in BES during peak PV generation time and supplied back during peak demand time at evening and can be considered as a part of future work.

\section{APPENDIX}

Line parameters for urban and rural cases are given in TABLE II and TABLE III respectively.

TABLE II. LINE PARAMETER FOR URBAN CASE

\begin{tabular}{|c|c|c|}
\hline Component & Parameter Value & Value for 20m \\
\hline $\mathrm{R}$ & $1.18 \mathrm{ohm} / \mathrm{km}$ & 0.0314 \\
\hline $\mathrm{X}$ & $.96 \mathrm{mH} / \mathrm{km}$ & 0.0314 \\
\hline
\end{tabular}

TABLE III. LINE PARAMETER FOR RURAL CASE

\begin{tabular}{|c|c|c|c|c|}
\hline \multirow{2}{*}{ Component } & \multicolumn{2}{|c|}{ Backbone Feeder } & \multicolumn{2}{c|}{ SWER Line } \\
\cline { 2 - 5 } & $\begin{array}{c}\text { Parameter } \\
\text { Value }\end{array}$ & $\begin{array}{c}\text { Value for } \\
\text { 20m }\end{array}$ & $\begin{array}{c}\text { Parameter } \\
\text { Value }\end{array}$ & $\begin{array}{c}\text { Value for } \\
\text { 100m }\end{array}$ \\
\hline $\mathrm{R}$ & $\begin{array}{c}1.18 \\
\mathrm{ohm} / \mathrm{km}\end{array}$ & 0.0314 & $\begin{array}{c}2.36 \\
\mathrm{ohm} / \mathrm{km}\end{array}$ & 0.314 \\
\hline $\mathrm{X}$ & $\begin{array}{c}.345 \\
\mathrm{mH} / \mathrm{km}\end{array}$ & 0.0113 & $\begin{array}{c}.345 \\
\mathrm{mH} / \mathrm{km}\end{array}$ & 0.0565 \\
\hline
\end{tabular}

\section{REFERENCES}

[1] AEMO report on "Rooftop PV Information Paper 2012", ABN 94072 010 327, ISSN: 1836-7593.

[2] P. McNutt, J. Hambrick, M. Keesee, and D. Brown, (2009). "Impact of SolarSmart Subdivisions on SMUD's Distribution System", Technical Report NRELTP-550- 46093. [Online] Available: http://www.nrel.gov/docs/fy09osti/46093.pdf

[3] T. Bopp et al., "Commercial and technical integration of distributed generation into distribution network", in CIRED Conf., Barcelona, Spain, May 12-15, 2003, pp. 1-4, Session 4, Paper 45.

[4] S. Cobben, B. Gaiddon, and H. Laukamp. (2008). "Impact of Photovoltaic Generation on Power Quality in Urban Areas with High PV Population", Intelligent Energy Europe/05/171/S12.420208, WP-4, Deliverable 4.3. [Online] Available: http://www.pvupscale.org/IMG/pdf/WP4_D4$\underline{3 \text { public v1c.pdf }}$
[5] F. Blaabjerg, Z. Chen, and S. B. Kjaer, "Power electronics as efficient interface in dispersed power generation systems", IEEE Trans. Power Electron., vol. 19, no. 5, pp. 1184-1194, Sep. 2004.

[6] Y. Ueda, K. Kurokawa, T. Tanabe, K. Kitamura, and H. Sugihara, "Analysis results of output power loss due to the grid voltage rise in gridconnected photovoltaic power generation systems," IEEE Trans. Ind. Electron., vol. 55, no. 7, pp. 2744-2751, Jul. 2008.

[7] V. Roberts, A. Beddoes, A. Colinson, and F. van Overbeeke, "Active networks for the accommodation of dispersed generation", in CIRED Conf., Barcelona, Spain, May 12-15, 2003, 1-6, Session 4, Paper 51.

[8] Review of Technical Options and Constraints for Integration of Distributed Generation in Electricity Networks [Online] Available: http://www.sustelnet.net

[9] Grid of the Future White Paper on Interconnection and Controls for Reliable, Large Scale Integration of Distributed Energy Resources [Online] Available: http://certs.lbl.gov

[10] C. L. Masters, "Voltage rise: the big issue when connecting embedded generation to long $11 \mathrm{KV}$ overhead lines," IEEE Power Eng. J., vol. 16, no. 1, pp. 5-12, Feb. 2002.

[11] T. Sansawatt, L. F. Ochoa, G.P. Harrison, "Smart Decentralized Control of DG for Voltage and Thermal Constraint Management", IEEE Trans. Power Syst., vol. pp, no. 99, pp. 1, 2012.

[12] L. Xiaoming, L. Qingfen, Y. Xianggen, and X. Jianghui, "A new on-load tap changing system with power electronic elements for power transformers", in Proc.2002 PowerCon, Int. Conf. on Power Syst. Technol., vol. 1, pp. 556-559.

[13] C. A. Coello, "An updated survey of evolutionary multiobjective optimization techniques: State of the art and future trends", in Proc. 1999 IEEE Congr. Evol. Comput., pp. 3-13.

[14] P. M. S. Carvalho, P. F. Correia, and L. A. F. Ferreira, "Distributed reactive power generation control for voltage rise mitigation in distribution networks", IEEE Trans. Power Syst., vol. 23, no. 2, pp. 766772, May 2008.

[15] F. A. Viawan, D. Karlsson, "Coordinate voltage and reactive powe control in the presence of distributed generation", IEEE PES General MeetingConvers. and Del. of Electrical Energy in the 21 $1^{\text {st }}$ Century, pp. 1-6, 2008.

[16] E. Demirok, P. Casado Gonzalez, K. H. B. Frederiksen, D. Sera, P. Rodriguez, and R. Teodorescu, "Local reactive Power Control Methods for Overvoltage Prevention of Distributed Solar Inverters in Low-Voltage Grids", IEEE J. of Photovolt., vol. 1, no. 2, pp. 174-182, 2011.

[17] J. Cappelle, J. Vanalme, S. Vispoel, T. Van Maerhem, B. Verhelst, C. Debruyne, and J. Desmet, "Introducing small storage capacity at residential PV installations to prevent overvoltages", IEEE Int. Conf. on Smart Grid Commun (SmartGridComm), pp. 534-539, 2011.

[18] C. Preve, "Protection of Electrical Networks", London: ISTE, 2006.

[19] H. Nicanfar, P. Jokar, K. Beznosov, V.C.M. Leung, "Efficient Authentication and Key Management Mechanisms for Smart Grid Communications," IEEE Systems Journal, vol., no.99, pp.1,12.

[20] W. Durbak and J. R. Stewart, "PLC Signal Attenuation in Branched Networks”, IEEE Trans. Power del., vol. 5, no. 2, pp. 878-883, Apr. 1990.

[21] G. Karady and X. Liu, "Fault management and protection of FREEDM systems," in Proc. 2010 IEEE Power Energy Soc. Gen. Meet.

[22] X. Lu, W. Wang, and J. Ma, "An Empirical Study of Communication, Infrastructures Towards the Smart Grid: Design, Implementation, and Evaluation", IEEE Trans. Smart Grid., vol. 4, no. 1, pp. 170-183, Mar. 2013.

[23] F. Shahnia, R. Majumder, A. Ghosh, G. Ledwich and F. zare, "Voltage imbalance analysis in residential low voltage distribution networks with rooftop PVs", Electric Power Systems Research, Vol. 81, No. 9, pp. 18051814, 2011.

[24] M. Molinas, J. A. Suul, and T. Undeland, "Low Voltage Ride Through of Wind Farms With Cage Generators: STATCOM Vs SVC", IEEE Trans. Power Elec. vol. 23, no. 3, May 2008.

Md Kabir is currently working for his $\mathrm{PhD}$ at Queensland University of Technology (QUT), Brisbane, Australia. His research area is grid integration of Photovoltaic in distribution systems.

Y. Mishra is a lecturer at QUT, Brisbane, Australia. His research interest includes smart grid applications in power system engineering. 